\title{
Leistungselektronik/Leistungshalbleiter - Anwendungen von Milliwatt bis Megawatt, vom Mikrochip bis zum System
}

\author{
B. Deutschmann
}

angenommen am 19. Jänner 2021, online publiziert am 27. Jänner 2021

(C) Springer-Verlag GmbH Austria, ein Teil von Springer Nature 2021

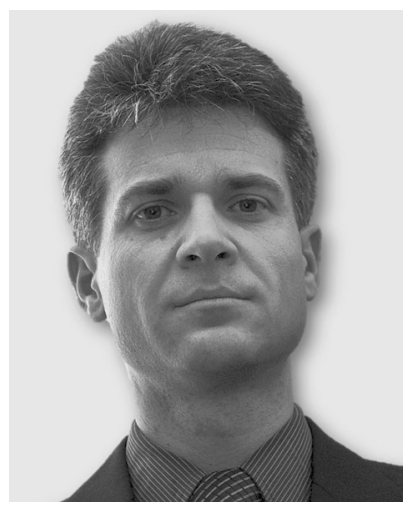

Univ.-Prof. Dipl.-Ing. Dr. techn. Bernd Deutschmann
Liebe Leserinnen, liebe Leser,

die moderne Elektronik sowie die Computer- und Telekommunikationstechnik haben unser Leben, die Art und Weise, wie wir arbeiten oder unsere Freizeit verbringen, und wie wir uns untereinander verständigen, grundlegend verändert. Eine Vielzahl von Erfindungen - vom einfachen Transistor oder dem integrierten Schaltkreis über Radio und Fernsehen bis hin zu Computern, Mobiltelefonen und GPS - war die Grundlage dafür, dass unser Leben heutzutage zunehmend von der Elektronik unterstützt (beherrscht) wird. In dieser technologiegetriebenen Welt stehen wir vor der Herausforderung, all unseren modernen technischen Innovationen die benötigte elektrische Energie verlustarm, umweltfreundlich, $\mathrm{CO}_{2}$-neutral und ressourcenschonend, aber vor allem auch in entsprechend angepasster Form, zum Beispiel als Wechselspannung oder als Gleichspannung, zur Verfügung zu stellen.

Hierzu liefert uns die Leistungselektronik seit vielen Jahren einen wertvollen Beitrag, denn keine dieser Innovationen wäre zustande gekommen, gäbe es da nicht die Technologie, die innen die nötige Energie dafür zur Verfügung stellt.

Das sollte Grund genug sein, die Leistungselektronik in der vorliegenden e\&i-Ausgabe einmal in das Rampenlicht zu stellen und aktuelle Forschungsprojekte auf diesem Gebiet zu beleuchten.

Was ist eigentlich Leistungselektronik?

Die Leistungselektronik, als ein immer wichtiger werdendes Teilgebiet der Elektrotechnik, beschäftigt sich in erster Linie mit der Umformung elektrischer Energie in Bezug auf deren Form, das heißt, die Höhe von Spannung und Strom sowie deren Kurvenform und Frequenz. Sie formt also die bereitgestellte elektrische Energie in die vom Verbraucher benötigte Form um. Diese Umformung erfolgt mittlerweile sehr effizient und verlustarm, zum Beispiel durch den Einsatz modernster Regelungstechnik und Mikroelektronik in Kombination mit innovativen Leistungshalbleitern aus Siliziumcarbid (SiC) oder Galliumnitrid (GaN).

Vielleicht lässt sich der Ursprung der auf Halbleitern basierenden Leistungselektronik mit der Erfindung einer Diode auf Siliziumbasis auf den Anfang des 20. Jahrhunderts (1906) zurückführen [1]. Mithilfe von Dioden war erstmals eine energieeffiziente und einfache Umformung von Wechselspannung in Gleichspannung möglich. Mitte des 20. Jahrhunderts war sicherlich die Erfindung des Bipolartransistors (Brattain, Bardeen und Shockley, 1947) der wichtigste
Meilenstein in der Geschichte der Elektronik [2, 3]. Im Gegensatz zur Elektronik war die Leistungselektronik in dieser Zeit sehr durch den Bedarf der Kontrolle größerer Ströme unter Verwendung von neuen Halbleitertechnologien geprägt. Dies ermöglichte erstmals die Erfindung des Thyristors basierend auf den Arbeiten von Shockley, Ebers und Moll [4]. 1957 wurde ein vierschichtiges p-n-p-n-Bauelement von General Electric (GE) als Silicon Controlled Rectifier (SCR, später Thyristor) eingeführt. Die ersten Thyristoren litten aber noch unter dem Nachteil, dass es einen erheblichen Aufwand bedurfte, diese wieder auszuschalten, wenn sie einmal eingeschaltet waren. Ein Umstand, der zum Beispiel durch die Erfindung des sogenannten Integrated Gate-Commutated Thyristor (GTO) wettgemacht wurde. Einer der wichtigsten Schritte im Bereich der Leistungselektronik in dieser Zeit war aber sicherlich die Entwicklung des Insulated Gate Bipolar Transistors (IGBT). Die Vorarbeiten dazu lieferten K. Yamagami und Y. Akagiri von Mitsubishi Electric, die 1968 in einer japanischen Patentanmeldung die Kombination eines MOS-Transistors, der ein PNPN-Halbleiterbauelement ansteuerte, vorschlugen [5, 6]. Darauf basierend entwickelte B. Jayant Baliga in den späten 70er-Jahren bei GE einen IGBT, der bis heute die moderne Leistungselektronik dominiert [7, 8]. Durch seine Vorteile in Bezug auf Zuverlässigkeit, Effizienz und Stromtragfähigkeit sowie die geringen Verluste sind IGBT-Module auch heute noch in einer Vielzahl von industriellen und automotiven Anwendungen bis hin in den Gigawattleistungsbereich zu finden.

Eine weitere wichtige Erfindung der Leistungselektronik ist unbestritten die des Super-Junction-Konzeptes in den 1980er-Jahren $[9,10]$. Dieses Konzept spielte eine fundamentale Rolle bei der Überwindung der bis zu diesem Zeitpunkt bekannten theoretischen Grenzen beim Einsatz des Halbleitermaterials Silizium, dem sogenannten Silicon-Limit [11]. Ein wesentliches Merkmal des SiliconLimits ist der Zusammenhang des Drain-Source-Durchlasswiderstands $\left(\mathrm{R}_{\mathrm{DS}}\right)$ im eingeschalteten Zustand mit der entsprechenden Durchbruchspannung $U_{B r}$ bei gegebener Chipfläche. Es gilt in diesem Zusammenhang, je höher die gewünschte maximale Sperrspannung des Leistungshalbleiterschalters, desto größer ist auch sein Durchlasswiderstand und damit einhergehend die Verluste. Das Ziel, geringe Verluste bei gleichzeitig hohen Durchbruchspannungen zu erreichen, war bis zur Entwicklung des Super-Junction-Konzeptes somit nicht möglich. Die technologische Realisierung dieses innovativen Konzeptes erfolgte dann Ende der 90er-Jahre mit der Vorstellung der ersten Produkte mit den Bezeichnungen "CoolMOS" (Infineon) bzw. „MDMesh" (ST Microelectronics), die in diesem Bereich bis heute eine Vorreiterrolle spielen $[12,13]$.

Deutschmann, Bernd, Institut für Elektronik, Technische Universität Graz, Inffeldgasse 12/I, 8010 Graz, Österreich (E-Mail: bernd.deutschmann@tugraz.at) 
An dieser Stelle darf ich schon einmal auf ein Interview in dieser e\&i-Ausgabe hinweisen, welches mit einem der Väter des "CoolMOS" , Hrn. Dr. Gerald Deboy, Sr. Principal bei Infineon Technologies Austria AG, geführt wurde.

Heute konkurrieren die auf Silizium basierenden Super-JunctionBauelemente mit den sogenannten Wide-Bandgap-Leistungshalbleiterbauelementen, die zum Beispiel auf Materialien wie Galliumnitrid ( $\mathrm{GaN}$ ) und Siliziumkarbid ( $\mathrm{SiC}$ ) basieren. Aufgrund des deutlich höheren Bandabstands dieser Halbleiter und den damit einhergehenden Vorteilen bezüglich höherer Schaltfrequenzen und besserer Stromtragfähigkeiten sowie höherer Temperaturen leiten sie aktuell eine neue Ära in der Geschichte der modernen Leistungselektronik ein. Mit ihnen kommen wir dem Ziel, Geräte zukünftig effizienter, kleiner und in Summe auch kostengünstiger zu realisieren, immer näher.

Grundlegende Kenntnisse auf dem Gebiet der Leistungselektronik werden heute in vielen Fachgebieten verstärkt benötigt, denn kaum ein Industriezweig kommt ohne die Vorteile leistungselektronischer Komponenten aus. Die Leistungselektronik hat sich zu einer Schlüsseltechnologie entwickelt, die in vielen Anwendungsfeldern wie zum Beispiel der Elektromobilität an Bedeutung gewinnt. Dies spiegelt sich auch in zwei Beiträgen zu diesem Thema in dieser e\&iAusgabe wider.

Im ersten Beitrag (Arash Pake Talei et al. „Considerations for a power line communication system for traction batteries") geht es um die Charakterisierung der Impedanz einer prismatischen LiIonen-Batteriezelle für den Einsatz in einer sogenannten Power Line Communication-Anwendung für ein Batteriemanagementsystem, wie es in Elektrofahrzeugen zum Einsatz kommt. Der zweite Artikel dazu (Madhavi Dhara et at. „Übertragungsfunktionen zur Abschätzung der gestrahlten Emissionen von HV-Kraftfahrzeugkabeln") behandelt einen systematischen Ansatz zur Modellierung der Übertragungsfunktion für zerlegte Gleich- und Gegentaktanregungen aus geometrischen Parametern und liefert ein analytisches Verständnis des Einflusses der Stromverteilungspfade in einem $\mathrm{Kfz}$ Elektroantrieb.

Traditionell ist die Leistungselektronik natürlich sehr stark im Bereich der Energieversorgung beheimatet. Inzwischen ist der Anteil der leistungselektronischen Geräte in netzgekoppelten Systemen der Energieversorgung bereits so groß, dass sie die dynamischen Eigenschaften des Energiesystems beeinflussen können. Wissenschaftliche Untersuchungen dazu zeigt der Beitrag von Ziqian Zhang et al. („Systematic stability analysis, evaluation and testing process, and platform for grid-connected power electronic equipment").

In den letzten Jahrzehnten zogen die Fortschritte der Mikroelektronik Schritt für Schritt auch in den Bereich der leistungselektronischen Bauelemente ein. Mit ihr gelang es, über ausgeklügelte Steuerungs- und Regelungsmöglichkeiten die Performance leistungselektronischer Anwendungen deutlich zu verbessern. Sollten Sie bis dato den Eindruck gewonnen haben, dass sich die Leistungselektronik vorwiegend mit Anwendungen im Mega- bzw. Gigawattbereich beschäftigt, dann wird Sie der Beitrag aus dem Bereich der Mikroelektronik von Stefan Schmickl et al. („Micro-watt power management: challenges of on-chip energy harvesting " wahrscheinlich eines Besseren belehren. Eine Vielzahl heutiger leistungselektronischer Anwendungen findet sich vorwiegend im Bereich der Mikroelektronik mit Leistungen im Micro-Watt-Bereich wieder. Gerade bei batterieunabhängigen loT-Geräten stehen wir zum Beispiel immer mehr vor der Herausforderung, diese möglichst energieeffizient mit integrierten Power Management- und Energy Harvesting-Systemen zu betreiben. Im Bereich der Mikroelektronik ist eines der vorrangigen Ziele fast immer die Reduzierung des Energieverbrauchs. Das zeigt auch der Beitrag von Markus Stadelmayer et al. („Low-power integrated transmitter design using frequency multiplication techniques - Edge-combining and third harmonic extraction"). Er behandelt den Entwurf von Transmittern mit extrem niedriger Leistung, die in der Lage sind, den Gesamtleistungsverbrauch durch den Einsatz von Frequenzvervielfachungstechniken zu reduzieren. Aber auch bei der Technologieentwicklung spielt die Forschung im Bereich der Leistungselektronik eine entscheidende Rolle. Im Beitrag von Osvaldo Gasparri et al. („Ultra-thin oxide breakdown for OTP development in power technologies") werden die Aspekte in Bezug auf den Oxiddurchbruch untersucht und auf die Entwicklung einer OTP-Zelle mit Antifuse-Funktion in einer 350nm-CMOS-Leistungstechnologie angewandt.

Um das elektrische Verhalten leistungselektronischer Produkte vorab bestimmen zu können, sind Simulationen mithilfe von Simulationsmodellen leistungselektronischer Komponenten wichtiger denn je. Ein weiterer Beitrag in dieser e\&i-Ausgabe (Michael Fuchs et al. "Fast and simple model generation for superjunction power MOSFETs") beschreibt dazu einen neuen Weg zur Erstellung eines Verhaltensmodells für Leistungs-MOSFETs mit hochgradig nichtlinearen parasitären Kapazitäten.

Ich möchte mich abschließend noch ganz herzlich bei den Autor/innen für die gewissenhafte Erstellung der vorliegenden Beiträge sowie bei den Gutachter/innen und der Redaktion für ihre professionelle Arbeit bedanken.

Ihnen, liebe Leserinnen und Leser, hoffe ich, mit diesem Schwerpunktheft zum Thema Leistungselektronik einen informativen Überblick zu geben. Ich freue mich sehr, wenn es mir gelungen ist aufzuzeigen, welche Entwicklungen und Forschungen es im Bereich der Leistungselektronik gibt und welche Anwendungen diese zukünftig ermöglichen werden.

Ich wünsche Ihnen viel Vergnügen beim Lesen der Beiträge!

Hinweis des Verlags Der Verlag bleibt in Hinblick auf geografische Zuordnungen und Gebietsbezeichnungen in veröffentlichten Karten und Institutsadressen neutral.

\section{Literatur}

1. Greenleaf Whittier Pickard (1906): Means for receiving intelligence communicated by electric waves. United States Patent US836,531, November 20.

2. Bardeen, J., Brattain, W. H. (1948): Semiconductor amplifier and electrode structure therefor. United States Patent US2,589,658, Original Application June 17, Serial No. 33,466 .

3. Bardeen, J., Brattain, W. H. (1950): Three-electrode circuit element utilizing semiconductor materials. United States Patent US2,524,035, Application June 17, 1948, Serial No. 33,466, patented Oct. 3.

4. Arsov, G. L., Mircevski, S. (2010): The sixth decade of the thyristor. J. Electron., 14(1), 3. ISSN 1450-5843.

5. Yamagami, K., Akagiri Transistor, Y. Japanese Patent No. S47-21739, File No. S4387175 .

6. Shenai, K. (2015): The invention and demonstration of the IGBT [a look back]. IEEE Power Energy Mag., 2(2), 12-16. https://doi.org/10.1109/MPEL.2015.2421751.

7. Baliga, B. J. (1977): MOS gate structure for thyristors. GE Patent Disclosure Letter RD-10 243, July 26

8. Baliga, B. J. (1979): Enhancement and depletion mode vertical channel MOS gated thyristors. Electron. Lett., 15, 645-647.

9. Tihanyi, J. (1995): Power MOSFET. United States Patent US5,438,215, Aug. 1.

10. Coe, D. J. (1988): High voltage semiconductor devices. United States Patent US4,754,310, Jun. 28

11. Deboy, G., März, M., Stengl, J.-P., Strack, H., Tihanyi, J., Weber, H. (1998): A new generation of high voltage MOSFETs breaks the limit line of silicon. In IEDM tech. dig. (S. 683-685).

12. Lorenz, L., Deboy, G., Knapp, A., Marz, M. (1999): COOLMOS—a new milestone in high voltage power MOS. In Proc. 11th int. symp. power semiconductor device ICS (S. 3-10).

13. Saggio, M., Fagone, D., Musumeci, S. (2000): MDmesh: innovative technology for high voltage power MOSFETs. In Proc. 12th int. symp. power semiconductor device ICs (S. 65-68). 